\title{
Characterisation of pleural inflammation occurring after primary spontaneous pneumothorax
}

\author{
A. De Smedt, E. Vanderlinden, C. Demanet, M. De Waele, A. Goossens, M. Noppen
}

\begin{abstract}
Characterisation of pleural inflammation occurring after primary spontaneous pneumothorax. A. De Smedt, E. Vanderlinden, C. Demanet, M. De Waele, A. Goossens, M. Noppen. (C) ERS Journals Ltd 2004.

ABSTRACT: The aim of this study was to examine the inflammatory reaction occurring in the pleural space of patients suffering from primary spontaneous pneumothorax (PSP) using pleural lavage, which was performed in patients with PSP and in healthy control subjects (essential hyperhidrosis patients undergoing thoracoscopy for sympathicolysis treatment). Cellular and solute composition of lavage fluid, peripheral blood and parietal pleural biopsies were analysed.

PSP lavage fluid showed an increase in all differentiated leucocytes, but most strikingly eosinophils and neutrophils. In the blood of patients with PSP, the total number of leucocytes and the absolute number of eosinophils, neutrophils and monocytes were also significantly increased. The time in which air was present in the pleural space was positively correlated with the increase of eosinophils in lavage fluid, parietal pleura and blood. Eosinophilic cationic protein was elevated after PSP and strongly correlated with the absolute number of lavage eosinophils. Chemo and cytokine analysis in lavage fluid showed differences in concentrations of interleukin (IL)-5, IL-6, IL-8, IL-12p40, tumour necrosis factor- $\alpha$ and RANTES, but not of eotaxin. Surprisingly, high levels of lipopolysaccharide binding protein were also measured.

Primary spontaneous pnumothorax is associated with a substantial pleural inflammatory reaction. The authors hypothesise that mechanical stretch factors, lipopolysaccharide binding protein/lipopolysaccharide complexes or other environmental components trigger pleural inflammation after primary spontaneous pnumothorax. Eur Respir J 2004; 23: 896-900.
\end{abstract}

Respiratory Division and HaematologyImmunology Dept, Academic Hospital, Brussels, Belgium.

Correspondence: M. Noppen, Respiratory Division, Academic Hospital, Academisch Ziekenhuis Vrije universiteit Brussel, 101 Laarbeeklaan, B-1090 Brussels, Belgium. Fax: 322477684

E-mail: marc.noppen@az.vub.ac.be

Keywords: Cytokines

eosinophils

inflammation

pleural lavage

pneumothorax

primary spontaneous.

Received: July 92003

Accepted after revision: February 112004

The Dept of Internal Medicine and the Respiratory Division of the Academic Hospital AZ-VUB supported this work
Primary spontaneous pneumothorax (PSP) is defined as the spontaneous occurrence of air in the pleural cavity in individuals without clinically apparent lung disease [1]. PSP occurs most often in male subjects with an asthenic body type. Smoking has also been strongly implicated in the pathogenesis of PSP [2]. Previous studies have demonstrated that inflammatory changes in bronchi, lungs and in the pleural cavity may be involved in the pathophysiology of PSP. Anatomical and histological changes in bronchi and lungs, e.g. emphysema-like changes [3] and chronic bronchiolitis $[4,5]$ have been observed in PSP, and ASKIN et al. [6] observed a reactive eosinophilic pleuritis in patients with PSP suggesting that air in the pleural space seems to be associated with an eosinophilic inflammatory response in the pleural space. Pleural effusions are rare, but pleural fluid eosinophilia (defined as $>10 \%$ of the total white cell count in the pleural fluid [7] has been observed in the majority of these cases [8]. SMIT et al. [9] observed that PSP causes a time-related increase of eosinophil cells in pleural fluid, which seemed to be mediated by interleukin (IL)-5. Usually, pleural fluid is not observed in patients with PSP. The authors have, therefore, developed a thoracoscopic method (pleural lavage) [10], to access the pleural space in a minimally invasive manner, and to allow analysis of the small volume of pleural fluid which is normally present [10].

The purpose of this study was to characterise the inflammatory changes occurring in the pleural space and the parietal pleura using thoracoscopic pleural lavage and parietal pleural biopsies in patients presenting with PSP, compared with otherwise healthy control subjects undergoing thoracoscopy for treatment of essential hyperhidrosis.

\section{Materials and methods}

\section{Patient population}

In total, 29 consecutive patients suffering from PSP were studied $(21$ males and eight females, with a mean \pm SD age of $36 \pm 16 \mathrm{yrs}$ ), and 30 healthy controls (nine male, 21 females, mean \pm SD $30 \pm 15$ yrs) who were referred for thoracoscopic treatment of severe essential hyperhidrosis. There were 21 smokers in the PSP group (72\%) and 10 smokers $(33 \%)$ in the control group $(\mathrm{p}=0.006)$. The time of onset of symptoms of pneumothorax was recorded and considered the start of air entry in the pleural space. A detailed history, physical examination, chest radiography and complete pulmonary function testing confirmed that all control subjects were in perfect health except for the presence of essential hyperhidrosis at the palmar and/or axillary level. All patients with PSP were also tested negative for any other lung disorder besides primary spontaneous pneumothorax before they were included. Verbal informed consent was obtained from every patient and the local ethics committee approved the study. 


\section{Pleural lavage technique}

The pleural lavage comprised of the injection and retrieval of $150 \mathrm{~mL}$ of saline from the pleural space prior to the talcage procedure in the patients with PSP, and prior to the thoracoscopic sympathicolysis procedure in the control population. All procedures were performed under strict aseptic conditions. More details concerning this technique are described elsewhere [10].

\section{Lavage fluid analysis}

A drop of well-mixed lavage fluid was introduced into a Bürker chamber for total leukocyte counting. The lavage fluid was centrifuged (10 min, 2,000 rpm) (TJ-6 Centrifuge, Beckman, Gent, Belgium) and $10 \mathrm{~mL}$ of the supernatant was removed and frozen at $-70^{\circ} \mathrm{C}$ for later analysis. The cell pellet was washed with $1 \mathrm{~mL}$ phosphate buffered saline (PBS)-1\% bovine serum albumin (BSA; Becton Dickinson, Erembodegem-Aalst, Belgium) and resuspended in PBS- $5 \%$ BSA until a suspension of $\sim 2 \times 10^{6}$ cells $\cdot \mathrm{mL}^{-1}$ was obtained. Cytospins were made with a cytocentrifuge (Cytospin 3, Shandon Scientific Ltd, Astmoor, UK) and stained with MayGrünwald-Giemsa. Differential cell counts were made on the stained slide, counting 300 cells by light microscopy $(\times 1,150)$.

The remaining lavage cells were suspended in one volume of PBS and nine volumes RNA-later (Sanbio, Uden, The Netherlands) for RNA preservation and frozen at $-70^{\circ} \mathrm{C}$ for RT-PCR analysis.

\section{Blood analysis}

Blood samples were taken on the same day as the pleural lavage. Total and differential counts of the peripheral blood cells were performed with an automatic cell counter (Cell-Dyn 4000, Abott, Louvain-la-Neuve, Belgium).

\section{Parietal pleura}

A total of five biopsies of the parietal pleura were obtained during thoracoscopy using $5 \mathrm{~mm}$ biopsy forceps. Specimens were fixed with Bouin and imbedded in paraffin. The biopsies were stained with haematoxylin-eosin-saffran and stained for myeloperoxidase and leukocyte cmmon antigen. The cells were counted with light microscopy per high powerfield, i.e. the number of cells on a field at a magnification of $\times 400$.

\section{Solute Analysis}

Eosinophilic cationic protein (ECP) concentrations were measured with a radio-immunoassay method (Pharmacia \&Upjohn, Brussels, Belgium) in the supernatant of unconcentrated lavage fluid. Commercially available ELISA kits were used for the quantification of: eotaxin (sensitivity test: $\left.<2.2 \mathrm{pg} \cdot \mathrm{mL}^{-1}\right)$, interferon- $\gamma\left(\right.$ IFN $\left.-\gamma ;<4 \mathrm{pg} \cdot \mathrm{mL}^{-1}\right)$, tumor necrosis factor- $\alpha$ (TNF- $\alpha$; $\left.1.7 \mathrm{pg} \cdot \mathrm{mL}^{-1}\right)$, interleukin (IL)-1 $\beta$ $\left(<1 \mathrm{pg} \cdot \mathrm{mL}^{-1}\right)$, RANTES $\left(<3 \mathrm{pg} \cdot \mathrm{mL}^{-1}\right)$, IL-2 $\left(0.1 \mathrm{U} \cdot \mathrm{mL}^{-1}\right)$, IL$5\left(<4 \mathrm{pg} \cdot \mathrm{mL}^{-1}\right)$, IL-6 $\left(5.5 \mathrm{pg} \cdot \mathrm{mL}^{-1}\right)$, IL-8 $\left(<5 \mathrm{pg} \cdot \mathrm{mL}^{-1}\right)$, bioactive IL-12 $\left(<0.8 \mathrm{pg} \cdot \mathrm{mL}^{-1}\right)$, IL-12p70 $\left(<4 \mathrm{pg} \cdot \mathrm{mL}^{-1}\right)($ Biosource, Nijvel, Belgium) and lypopolysaccharides (LPS)binding protein (LBP) $\left(1 \mathrm{ng} \cdot \mathrm{mL}^{-1}\right.$; Sanbio, Uden, Holland; LPS from Serratia Marcescens $\left(100 \mathrm{pg} \cdot \mathrm{mL}^{-1}\right.$; Sigma-Aldrich chemie, Schnelldorf, Germany) were measured as described by Peterfi AND Kocsis [11].

\section{Microbiological analysis}

Samples of the lavage fluid were tested for the presence of bacteria and fungi.

\section{Statistical Analysis}

Cellular data are expressed as median and interquartile ranges (IR: P25-P75). Comparison between data for the PSP and the control population were made with the nonparametric two-sided Mann-Whitney test. Correlations between data were analysed with the Spearman-correlation coefficient (r). Significance was accepted at $\mathrm{p}<0.05$.

\section{Results}

All lavage procedures were uneventful. Lavage prolonged the thoracoscopy time by only $1-3$ mins and no complications were observed.

\section{Cell counts in lavage fluid and peripheral blood}

Lavage fluid. The authors observed a significant increase in the absolute number of all nucleated cells after PSP. Relative differential cell counts in patients with PSP versus the controls are shown in figure 1 , and absolute cell counts in table 1 . Whereas the percentages of lymphocytes and macrophages predominate in normal control subjects [10], neutrophils and eosinophils were increased in PSP at the expense of lymphocytes and macrophages. The percentage of mesothelial cells was not significantly different in PSP as compared with normal controls.

Peripheral blood. The white blood cell (WBC) differential counts in the peripheral blood of patients with PSP and healthy controls are presented in table 2. There was a significant increase in total WBC count and in neutrophils, eosinophils

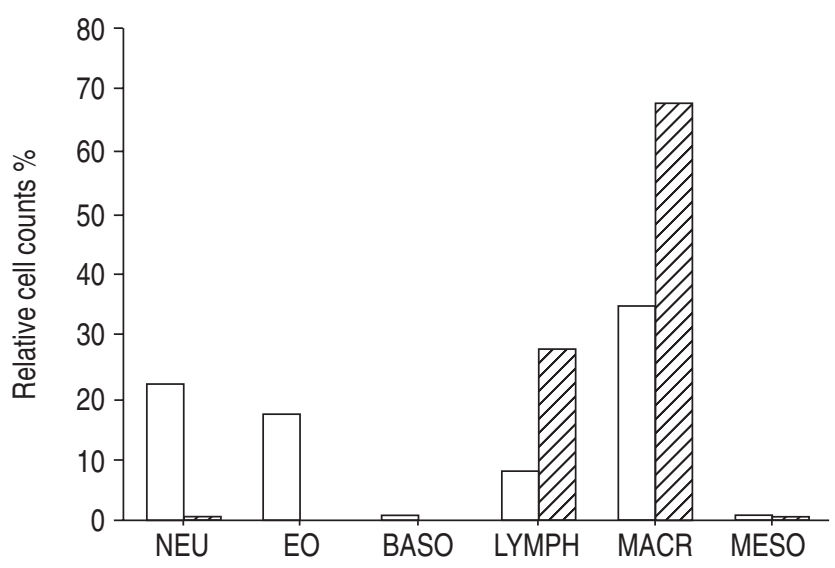

Fig. 1. - Lavage cell counts. Absolute cell counts per $\mathrm{mm}^{3}$ in lavage fluid of primary spontaneous pneumothorax patients $(\square)$ and controls $(\mathbb{C})$ are expressed as median (interquartile range: 25-75). Differential cell counts: distinct neutrophils (NEU), eosinophils (EO), basophils (BASO), lymphocytes (LYMPH), macrophages (MACR) and mesothelial cells (MESO). 
Table 1. - Lavage cell counts

\begin{tabular}{lccr}
\hline & PSP per $\mathrm{mm}^{3}$ & CONTR per $\mathrm{mm}^{3}$ & p-value \\
\hline WBC & $1973(547-3040)$ & $159(74-214)$ & $<0.001$ \\
NEU & $384(59-1052)$ & $1(0-2)$ & $<0.001$ \\
EO & $243(120-635)$ & $0(0-0)$ & $<0.001$ \\
BASO & $22(3-48)$ & $0(0-0)$ & $<0.001$ \\
LYMPH & $102(46-201)$ & $27(14-60)$ & 0.024 \\
MACR & $671(268-919)$ & $118(47-146)$ & $<0.004$ \\
MESO & $10(0-35)$ & $0(0-2)$ & 0.034
\end{tabular}

Absolute cell counts per $\mathrm{mm}^{3}$ in lavage fluid of primary spontaneous pneumothorax (PSP) patients and controls (CONTR) are expressed as median (interquartile range: 25-75). WBC: white blood cell counts; NEU: neutrophils; EO: eosinophils; BASO: basophils; LYMPH: lymphocytes; MACR: macrophages; MESO: mesothelial cells.

and monocytes in patients with PSP. A correlation was found between the absolute number of eosinophils in the lavage fluid and in the blood $(\mathrm{r}=0.61 ; \mathrm{p}=0.002)$.

\section{Duration of the pneumothorax}

The duration of the pneumothorax (expressed as the number of hours between the occurrence of symptoms and the thoracoscopic intervention) correlated with the observed changes in differential cell counts. The relative number of eosinophils correlated with the time of air presence in the pleural space $(\mathrm{r}=0.85, \mathrm{p}<0.001)$.

\section{Inflammation in the parietal pleura}

During the thoracoscopic intervention, blebs were occasionally present in the PSP population. Signs of rupture or bleeding were not found. Inflammatory changes in the parietal pleura of patients with PSP were invariably observed. The haematoxyline-eosine-saffran staining clearly showed the presence of eosinophils, and both mesothelial thickening and proliferation of the pleura. The staining for myeloperoxidase and leukocyte common antigen respectively showed an increase in neutrophils, and all WBC in the parietal pleura. No eosinophils or neutrophils were present in the parietal pleura of healthy controls. In patients with PSP however, there were $1-34$ (median 6) eosinophils $(\mathrm{p}=0.002)$ and 6-12 (median 7) neutrophils $(\mathrm{p}<0.001)$ per high power field. The number of eosinophils in the parietal pleura correlated with the number of eosinophils in the lavage fluid $(r=0.76$, $\mathrm{p}=0.002$ ).

\section{Eosinophil activation}

The concentration of eosinophilic cationic protein (ECP) in the lavage fluid of patients with PSP was increased (median: 19; IR: 9-43) $\mu \mathrm{g} \cdot \mathrm{L}^{-1}$, whereas in the control group ECP concentrations were not detectable $\left(<2 \mu \mathrm{g} \cdot \mathrm{L}^{-1}\right)$. The ECP concentration and the absolute number of eosinophils in the lavage fluid were strongly correlated $(r=0.96, p<0.001)$.

\section{Concentration of cytokines, chemokines and other biomolecules in pleural fluid supernatant}

The concentration of several chemokines and cytokines was measured in the lavage fluid of patients with PSP and in the control group. The concentrations of IL-5, IL-6, IL-8, TNF- $\alpha$
Table 2.-Blood cell counts

\begin{tabular}{lccc}
\hline & PSP per $\mathrm{mm}^{3}$ & CONTR per $\mathrm{mm}^{3}$ & p-value \\
\hline WBC & $9750(7130-11950)$ & $7800(5700-8600)$ & 0.016 \\
NEU & $5259(4494-7337)$ & $4032(3470-4948)$ & 0.016 \\
EO & $255(130-344)$ & $90(79-111)$ & 0.003 \\
BASO & $0(0-83)$ & $58(22-80)$ & NS \\
LYMPH & $2344(1679-2791)$ & $2520(1752-2708)$ & NS \\
MONO & $765(630-895)$ & $430(384-578)$ & $<0.001$ \\
\hline
\end{tabular}

Absolute cell counts per $\mathrm{mm}^{3}$ in lavage fluid of primary spontaneous pneumothorax (PSP) patients and controls (CONTR) are expressed as median (interquartile range: 25-75). WBC: white blood cell counts; NEU: neutrophils; EO: eosinophils; BASO: basophils; LYMPH: lymphocytes; MONO: monocytes; MESO: mesothelial cells; NS: not significant.

and LBP were significantly increased after PSP (fig. 2), whereas RANTES was decreased . A weak, but significant positive correlation was found between TNF- $\alpha /$ IL- 6 and TNF- $\alpha /$ IL-8 $(p=0.04 ; r=0.41$ and $p=0.03 ; r=0.43)$. IL-12 is a $70 \mathrm{kDa}(\mathrm{p} 70)$ lymphokine composed of a subunit of $40 \mathrm{kDa}$ (p40) and a subunit of $35 \mathrm{kDa}$ (p35) linked through a disulphide bridge. The concentration of IL-12 (p40+p70) was increased in the lavage fluid of PSP patients (median: 23.9; IR: 8.3-43.6) in comparison with the control group (median: 8.9; IR: 6.2-9.9), but no increase was found for IL-12 (p70), suggesting that only the p40 subunit was present. The concentrations of IFN- $\gamma$, IL- $1 \beta$, IL-2, eotaxin and LPS, showed no significant differences (data not shown). However, a significant difference $(\mathrm{p}=0.03)$ in LPS concentration was found between the smokers in both populations. The concentration of LBP $\left(\mathrm{ng} \cdot \mathrm{mL}^{-1}\right)$ was higher in the PSP population (median: 97.3; IR: 91.8-101.2) in comparison to the control group (median: 0.7; IR: 0.4-1).

\section{Microbiological analysis}

All sample cultures of healthy controls and patients with PSP were negative for bacteria and fungi.

\section{Discussion}

This is the first prospective study on thoracoscopic pleural lavage, pleural biopsy, and peripheral blood findings in consecutive patients with PSP, compared with healthy control subjects. This study shows a substantial pleural inflammatory reaction in patients with PSP, which is characterised by increases in pleural fluid and parietal pleural tissue eosinophils and neutrophils, and associated with a characteristic cytokine pattern.

Only two reports on pleural cell counts in patients with PSP have previously been published $[8,9]$,showing increases in total and differential cell counts which are comparable with the results in our patients with PSP. The present findings confirm that PSP is associated with a dramatic influx of eosinophils, but also of neutrophils in the pleural space. Our results also confirm the previously demonstrated relationship between the number of eosinophils in the pleural fluid and the duration of the pneumothorax.

Inflammatory cellular changes in the peripheral blood occurring after PSP were also examined for. Two case reports [8] have described the blood cell counts after PSP associated with pleural effusions, yielding 8,200 and $11,300 \mathrm{WBC} \cdot \mathrm{mm}^{-3}$, respectively with $5 \%$ and $3 \%$ eosinophils, $70 \%$ and $80 \%$ neutrophils, $23 \%$ and $16 \%$ lymphocytes and $2 \%$ and $1 \%$ monocytes, but no comparison with control subjects was 

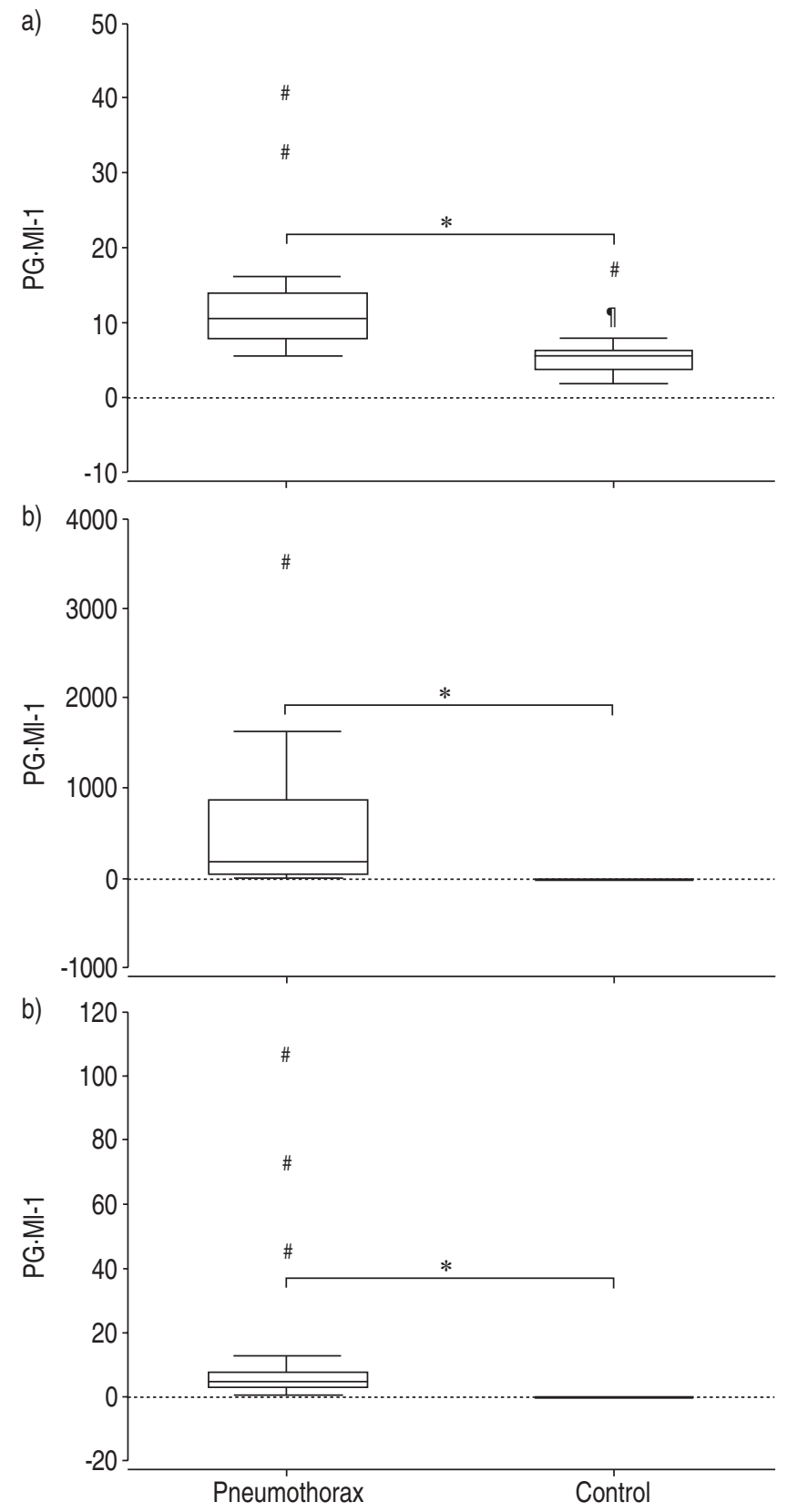

Fig. 2. - Data a) tumour necrosis factor- $\alpha$, b) interleukin IL)-6 and c) IL-8 concentration in pleural lavage fluids of patients with primary spontaneous pneumothorax and controls, presented as $\mathrm{pg} \cdot \mathrm{mL}^{-1}$ as median, (interquartile range 25-75), minimum and maximum. \#: extremes; : outliners; *: $\mathrm{p}<0.05$.

made. The authors observed, in comparison with normal subjects, a significant increase of the total number of WBC (9, 750 versus $\left.7,800 \cdot \mathrm{mm}^{-3}\right)$, eosinophils $\left(255\right.$ versus $\left.90 \cdot \mathrm{mm}^{-3}\right)$, neutrophils $\left(5,259\right.$ versus. $\left.4,032 \cdot \mathrm{mm}^{-3}\right)$ and monocytes $(765$ versus $430 \cdot \mathrm{mm}^{-3}$ ) in the peripheral blood of patients wih PSP. A correlation between the absolute number of eosinophils in the blood and lavage fluid was also present.

Increased numbers of eosinophils and neutrophils in the parietal pleura have also been described [6, 7], but no quantitative data are available. The authors observed no eosinophils or neutrophils in the parietal pleura of the control group, whereas these inflammatory cells were abundantly present in the parietal pleura after PSP. Furthermore, the number of eosinophils in the parietal pleura correlated with the increased number of eosinophils in the lavage fluid in patients with PSP.

ECP, a marker for eosinophil activation [12, 13] was not detectable in the control subjects $\left(<2 \mu \mathrm{g} \cdot \mathrm{L}^{-1}\right)$, whereas in the patients with PSP increased ECP concentrations were observed (median: 19; IR: 9-43 $\mu \mathrm{g} \cdot \mathrm{L}^{-1}$ ). ECP concentration correlated well with the absolute number of eosinophils in the pleural lavage fluid, suggesting activated eosinophils to be the source of ECP.

The exact mechanism of pleural inflammation occurring after PSP is unclear. IL-5, an eosinophil-specific cytokine stimulating chemoattraction and activation of eosinophils [12, $13,14]$ may play a key role in this inflammatory process $[9,15$, present study] whereas other eosinophil-specific cytokines and chemokines (e.g. eotaxin, RANTES) seem not to be involved. The observations from this current study, of increased numbers of neutrophils in the pleural lavage fluid and of an increased IL- 8 concentration, also point to a more nonspecific component of inflammatory reaction. PENIDO et al. [16] have reported that migration of eosinophils into the pleural space can occur independently of eotaxin, RANTES and IL-5 after intrapleural stimulation with LPS. LPS, a component of bacterial endotoxin, is a potent inducer of cell activation and induces secretion of a large number of pro-inflammatory cytokines $[16,17]$. In the presence of LPS-induced inflammation, respiratory epithelial cells produce LBP, an acute phase protein and most important defender against bacterial endotoxins [18]. LBP forms complexes with LPS, to which macrophages respond by producing TNF- $\alpha$ and IL-1. Following release of these cytokines, an inflammatory cascade is initiated including the production of IL-6 and IL-8, in turn resulting in increased LBP production [19]. Although LPS concentrations were not different in the lavage fluids of controls and patients with PSP, there was a significant increase after PSP as compared with controls in the actively smoking subjects, and LBP concentrations were also significantly higher in all PSP subjects. The authors therefore hypothesise, in accordance with previous observations, that PSP-induced exposure of the pleural space to environmental air could lead to an inflammatory reaction (either induced by mechanical stretching of the pleura, or by pro-inflammatory effects of one or more contaminants such as LPS or tobacco smoke), which is characterised by the influx of eosinophils [16] and by the production of LBP [18]. The former may stimulate IL-5 production [20], whereas the latter may stimulate the production of TNF- $\alpha$, IL-1, IL-6, and IL-8. Alternatively, as all but two patients were treated with chest tube drainage prior to thoracoscopy, the presence of this foreign body in the pleural space may also have contributed to the observed inflammatory changes. In order to exclude this hypothesis, PSP patients should have undergone thoracoscopy without previous aspiration chest tube drainage. However, this occurs only rarely in clinical practice and was performed in only two patients with PSP. Interestingly, pleural and blood analyses in these patients were similar to those in chest-tube treated patients.

Not unexpectedly, there were more smokers in the PSP population. Cigarette smoking undoubtedly is involved in the pathogenesis of PSP [2], however, its role in the pleural inflammation occurring after PSP, the subject of this study, is unclear. The observation that LPS concentrations are higher in the pleural lavage fluid of smoking patients with PSP as compared with smoking, healthy controls, and the fact that cigarette smoke is a known source of LPS [21], may support the hypothesis explained above.

The authors conclude that primary spontaneous pneumothorax is associated with a substantial pleural inflammatory reaction, which is characterised by the influx (and 
activation) of eosinophils and neutrophils into the pleural space and parietal pleura, and by an increase in the total white blood cell count in the peripheral blood. Interleukin-5, interleukin-6, interleukin 8, tumour necrosis factor- $\alpha$, interleukin-12p40 and lipopolysaccharide binding protein are significantly increased in the pleural lavage fluid. This inflammatory pattern may have been induced by a lipopolysaccharide-mediated defense response.

Acknowledgements. The authors would like to thank the personnel of the haematology/immunology and microbiology laboratories.

\section{References}

1. Schramel FMNH, Postmus PE, Vanderschueren RGJRA. Current aspects of spontaneous pneumothorax. Eur Respir $J$ 1997; 10: 1372-1379.

2. Deenstra M. Pneumothorax, Longziekten 1999; 743-747.

3. Ohata M, Suzuki H. Pathogenesis of spontaneous pneumothorax. Chest 1980; 77: 771-776.

4. Cottin V, Streichenberger N, Gamondes JP, Thévenet F, Loire R, Cordier JF. Respiratory bronchiolitis in smokers with spontaneous pneumothorax. Eur Respir $J$ 1998; 12: 702-704.

5. Lichter I, Gwynne JF. Spontaneous pneumothorax in young subjects. Thorax 1971; 26: 409-417.

6. Askin FB, McCann BG, Kuhn C. Reactive eosinophilic pleuritis. Arch Pathol Lab Med 1977; 101: 187-191.

7. Adelman M, Abelda SM, Gottlieb J, Haponik EF. Diagnostic utility of pleural fluid eosinophilia. Am J Med 1984; 77: 915-920.

8. Saini JS, Lal M, Rai J. Eosinophilic pleural effusion following pneumothorax. Indian $J$ Chest Dis Allied Sci 1980; 22: 133-136.

9. Smit HJM, Van Den Heuvel MM, Barbierato SB, Beelen $\mathrm{RJH}$, Postmus PE. Analysis of pleural fluid in idiopathic spontaneous pneumothorax; correlation of eosinophil percentage with the duration of air in the pleural space. Respir Med 1999; 93: 262-267.

10. Noppen M, De Waele M, Li R, et al. Volume and cellular content of normal pleural fluid in humans examined by pleural lavage. Am J Respir Crit Care Med 2000; 162: 1023 1026.

11. Peterfi Z, Kocsis B. Comparison of blocking agents for an ELISA for LPS. $J$ immunoassay 2000; 21: 341-54.

12. Rothenberg ME. Eosinophilia. New Engl J Med 1998; 338: $1592-1600$.

13. Weller PF. The immunobiology of eosinophils. New Engl $J$ Med 1991; 324: 1110-1118.

14. Schandené L, Namias B, Crusiaux A, et al. IL-5 in posttraumatic eosinophilic pleural effusion. Clin Exp Immunol 1993; 93: 115-119.

15. Gleich GJ. Mechanisms of eosinophil-associated inflammation. J Allergy Clin Immunol 2000; 105: 651-663.

16. Penido C, Castro-Faria-Neto HC, Vieira-de-Abreu A, et al. LPS induces eosinophil migration via CCR3 signaling through a mechanism independent of RANTES and Eotaxin. Am J Respir Cell Mol Biol 2001; 25: 707-716.

17. Bozza PT. Requirement for lymphocytes and resident macrophages in LPS-induced pleural eosinophil accumulation. J Leukoc Biol 1994; 56: 151-158.

18. Dentener M, Vreugdenhil A, Hoet P, et al. Production of the acute-phase protein lipopolysaccharide-binding protein by respiratory type II epithelial cells. Am J Respir Cell Mol Biol 2000; 23: 146-153.

19. Martin T. Overview of cytokine networks in lung injury. New York, American Thoracic Society Publication, 1999; 2 : 19-27.

20. Nakamura $\mathrm{Y}$, Ozaki $\mathrm{T}$, Kamei $\mathrm{T}$, et al. Factors that stimulate the proliferation and survival of eosinophils in eosinophilic pleural effusion: relationship to granulocyte/ macrophage colony-stimulating factor, interleukin-5, interleukin-3. Am J Respir Cell Mol Biol 1993; 8: 605-611.

21. Hasday JD, Bascom R, Costa JJ, Fitzgerald T, Dubin W Bacterial endotoxin is an active component of cigarette smoke. Chest 1999; 115: 829-835. 